\title{
Error-Bounds for the Evaluation of Integrals by the Euler-Maclaurin Formula and by Gauss-Type Formulae
}

\author{
By John McNamee
}

1. Introduction. This paper originated in an attempt to generalize a result of Goodwin's [1]. Goodwin showed that integrals of the type

$$
\int_{0}^{\infty} e^{-x^{2}} f(x) d x
$$

where $f(x)$ is even, can be evaluated with surprising accuracy by means of the trapezoidal-sum formula. It is natural to anticipate that a more general result can be obtained when the integrand is not restricted to the form given above; the generalization was easily obtained by contour integration. The guiding idea which we employ in obtaining error-bounds is extremely simple: we express the error-term of an approximate integration by a contour integral and then choose a contour (among those which enclose the singularities of the integrand) on which the error-term is simply and easily bounded. Most often, it turns out that minimizing the absolute value of the error-term integrand is quite effective; and, in general, we have preferred methods for obtaining error-bounds which can be used and extended without undue expenditure of time by the staff of a computing laboratory. Subsequently, it was found that the technique of minimizing the integrand had been used earlier by Davis [2] in a paper whose contents somewhat overlap the contents of the present paper. Further trial showed that the guiding idea could be used to obtain useful error-bounds for Gauss-type formulae. These bounds are reported in Section 3. In the main, the paper is concerned with the Euler-Maclaurin formula and extensions of it.

Examination of the literature showed that numerical techniques for evaluation of infinite integrals by means of equi-spaced ordinates of the integrand are closely related to a body of classical work on the Euler-Maclaurin formula. Abundant references will be found in the Enzyklopadie der Mathematischen Wissenschaften (Band I A 3 §38, p. 102-105, E §11 p. 929-931, Band II A 12 §105, p. 1324-1337, C 2 $\$ 9$, p. 91-96, C 7, \$10, p. 711-716) and useful summaries are given in Lindelöf [3] and in Hardy [4]. A summary of current methods for the evaluation of integrals may be found in the second edition of Modern Computing Methods [5].

The Euler-Maclaurin formula is commonly derived from real-variable theory, and it is fairly generally accepted that, in this form, the formula is of limited use in numerical integration. Our experience in the present investigation is that contour integration-where it is applicable-provides an easier route to the comprehension and efficient use of the formula. The conditions for the validity of the contour integral and for the bound of the error bear on the behaviour of the integrand in the complex plane; in real-variable theory, the conditions bear on the derivatives of the

Received November 12, 1963. Part of this work was done when the author was a guest worker at the National Bureau of Standards in the summer of 1961. 
integrand. A bound on the value of a complex integrand is often more easily obtained than a bound on a derivative of moderate or high order of a real integrand.

It may be useful to state at once the main results which are obtained here for evaluation of integrals by equi-spaced ordinates. The contour integral method expresses an integral as a sum of three terms: a summation (in general, trapezoidal or mid-ordinate), a correction term, and an error term. Assuming for the moment that the error term can be suitably bounded, the following points are easily demonstrated.

(i) The error term vanishes for a limited class of integrands. A similar point had been noted earlier by Fettis [6]; but it is perhaps more important to note that realvariable analysis and complex-variable analysis of the Euler-Maclaurin formula are not completely equivalent save for this limited class of integrands.

(ii) The correction term is zero if the integrand is even; the accuracy of the trapezoidal sum is then bounded only by the magnitude of the error term.

(iii) When the integrand is not even, it is possible to evaluate the integral by forming a sequence of sums using decreasing tabular intervals $h_{1}, h_{2}, \cdots, h_{m}$, the successive summations extending over smaller effective ranges. The accuracy obtainable is of course bounded by the sum of the successive error terms; if the successive error terms can be made to vanish, the accuracy is comparable with the accuracy of a single summation using the smallest interval $h_{m}$ but is much more economical of ordinates.

(iv) The device described in (iii) may be used when the integrand has a simple type of singularity at the origin, e.g. $x^{a}(a>-1)$ or $\log x$. In general, however, it is preferable to eliminate the singularity by a change of variable when the change can conveniently be made.

2. Evaluation of Complete Semi-infinite Integrals. We shall be concerned almost exclusively with complete semi-infinite integrals, i.e. integrals over the range $(0, \infty)$. The analysis for incomplete integrals, i.e. over the range $\left(x_{0}, \infty\right)$-supposing a change of variable to be inexpedient-or for finite integrals, is similar, but somewhat more complicated since it requires parameters specifying the ends of the range.

2.1. Rectangular Contour Integral. The classical rectangular-contour analysis provides a generalization which includes Goodwin's result. Lindelöf (1905) states that the application of this contour in the summation of series is due in essence to Cauchy [7] though the connection with the Euler-Maclaurin expansion was known to Plana [8] and to Abel [9]. It will be necessary to state the classical analysis briefly in order to emphasize our departure from the classical interest in summation. To anticipate a little we may characterize the departure by saying that quite weak conditions are tolerable if we are aiming only at a limited accuracy of evaluation.

In order to obtain an approximation to the integral

$$
\int_{0}^{\infty} g(x) d x
$$

we integrate the function

$$
\pi g(z) \cot \pi(z / h-\lambda)
$$

round the rectangular contour $L$ bounded by the lines $y= \pm d, x=+R$, and allow $R$ to approach infinity by finite steps which avoid the poles of the function on the 
real axis. The parameters are defined thus: $\lambda$ is a real parameter in the range $0 \leqq \lambda<1, h$ is to be the tabular interval of the summation approximation and $d$ is the half-height of the rectangle; $d$ is at present unrestricted though we may be obliged later to limit its magnitude.

We suppose that $g(z)$ is analytic and single-valued within the contour and that the integral

$$
I=\int_{0}^{\infty} g(x) d x
$$

exists (it is not difficult to extend the analysis to an integrand which is meromorphic within the contour). We shall also require that

$$
\lim _{R \rightarrow \infty} \int_{-d}^{d} e^{-2 \pi|y| / h} g(R+i y) d y=0
$$

but, when $d$ is finite, (2) can be replaced by the simpler, and stronger, condition

$$
g(R \pm i y) \rightarrow 0
$$

uniformly with respect to $y, R \rightarrow \infty$, when $-d \leqq y \leqq d$.

We have from the theory of residues

$$
\begin{aligned}
& \frac{1}{2 \pi i} \int_{L} \pi g(z) \cot \pi(z / h-\lambda) d z \\
& \quad=h[\epsilon g(\lambda h)+g(h+\lambda h)+g(2 h+\lambda h)+\cdots]
\end{aligned}
$$

where

$$
\epsilon= \begin{cases}1, & \lambda>0 \\ \frac{1}{2}, & \lambda=0,\end{cases}
$$

and the sum (3) embraces the residues at the poles of $\cot \pi(z / h-\lambda)$ within $L$.

The integral on the left-hand side of (3) can be simplified by a well-known device. We may write

$$
\frac{1}{2 i} \cot \pi\left(\frac{z}{h}-\lambda\right)=-\frac{1}{2}-\frac{e^{2 \pi i(z / h-\lambda)}}{1-e^{2 \pi i(z / h-\lambda)}}
$$

on the upper half of the contour; and

$$
\frac{1}{2 i} \cot \pi\left(\frac{z}{h}-\lambda\right)=\frac{1}{2}+\frac{e^{-2 \pi i(z / h-\lambda)}}{1-e^{-2 \pi i(z / h-\lambda)}}
$$

on the lower half of the contour (this device ensures that the second term on the right of these expressions is $O(\exp (-2 \pi|y| / h))$. Since $g(z)$ is assumed to be analytic in the closed domain, it is easy to show that

$$
\int_{0}^{\infty} g(x) d x-T(\lambda, h)=E(\lambda, h, d)+C(\lambda, h, d)
$$

where $T(\lambda, h)=h[\epsilon g(\lambda h)+g(h+\lambda h)+\cdots]$ and the integral is thus expressed

\footnotetext{
* See e.g. Lindelöf [3, p. 55-56], Hardy [4, p. 339-341].
} 
as a sum of three terms. Here, $\epsilon$ is defined in (3), and $E, C$, are defined by

$$
\begin{aligned}
& E(\lambda, h, d)=-e^{-2 \pi d / h} \int_{0}^{\infty}\left[\frac{g(x+i d) \exp 2 \pi i(x / h-\lambda)}{1-\exp 2 \pi[-d / h+i(x / h-\lambda)]}+\cdots\right] d x \\
& \cdots \text { denoting a conjugate term; } \\
& C(\lambda, h, d)=-\frac{i}{2} \int_{0}^{d}[g(i y)-g(-i y)] \frac{\cos 2 \pi \lambda-\exp -(2 \pi y / h)}{\cosh 2 \pi y / h-\cos 2 \pi \lambda} d y \\
&-\frac{\sin 2 \pi \lambda}{2} \int_{0}^{d} \frac{g(i y)+g(-i y)}{\cosh 2 \pi y / h-\cos 2 \pi \lambda} d y
\end{aligned}
$$

The first term on the right of (4) can be regarded as an error term, provided that it is possible by a judicious choice of $h$ and $d$ to arrange that the damping factor $\exp (-2 \pi d / h)$ is sufficiently powerful to make $E$ negligible for a prescribed precision of evaluation. The second term, $C$, may be termed a correction term: it corrects the trapezoidal sum and must in general be evaluated numerically. The correction term assumes a simpler and more familiar form if we take $\lambda=0, \frac{1}{2}$. We have then

$$
C(0)=-i \int_{0}^{d} \frac{g(i y)-g(-i y)}{\exp (2 \pi y / h)-1} d y, \quad C\left(\frac{1}{2}\right)=i \int_{0}^{d} \frac{g(i y)-g(-i y)}{\exp (2 \pi y / h)+1} d y .
$$

If the numerators in these integrals are expressed as Taylor series, we obtain expansions which are analogous to the Euler-Maclaurin expansion and become formally identical with it when $d$ approaches infinity. ${ }^{*}$ More generally, we can write $C(\lambda, h, d)$ as a Taylor expansion and obtain

$$
\int_{0}^{\infty} g(x) d x-T(\lambda, h)=\sum_{1}^{m} \frac{B_{n}{ }^{\prime}(\lambda)}{n !} h^{n} g^{(n-1)}(0)+R_{m}+E(\lambda, h, d)
$$

where $R_{m}$ is the truncation error incurred in replacing the correction term by the finite summation on the right of $(7)$ and the coefficients $B_{n}{ }^{\prime}(\lambda)$ are defined by

$$
\begin{gathered}
B_{2 n}^{\prime}(\lambda)=(-)^{n+1} 4 n \int_{0}^{d / h} y^{2 n-1} \frac{\cos 2 \pi \lambda-\exp (-2 \pi y)}{2[\cosh 2 \pi y-\cos 2 \pi \lambda]} d y, \quad n=1,2, \cdots . \\
B_{2 n+1}^{\prime}(\lambda)=(-)^{n+1}(4 n+2) \int_{0}^{d / h} y^{2 n} \frac{\sin 2 \pi \lambda}{2[\cosh 2 \pi y-\cos 2 \pi \lambda]} d y, \quad n=0,1,2, \cdots .
\end{gathered}
$$

The limit of $B_{n}{ }^{\prime}(\lambda)$, as $d \rightarrow \infty$, is the Bernoulli polynomial $B_{n}(\lambda)$ and the limit of $B_{n}{ }^{\prime}(0)$ is the Bernoulli number of order $n$ (we follow the notation of Nörlund and Milne-Thomson for the Bernoulli polynomials). The identification of (7) in the limit $d \rightarrow \infty$ with the generalized form of the Euler-Maclaurin formula is valid when the term $E(\lambda, h, \infty)$ is zero. A sufficient condition for the vanishing of $E$ is

$$
e^{-2 \pi|y| / h}|g(x \pm i y)| \rightarrow 0
$$

uniformly, $y \rightarrow \infty$, in the interval $0 \leqq x \leqq \infty$. In addition, the validity of the contour integration requires that (2) must hold in the limit $d \rightarrow \infty$.

These conditions would unduly restrict the class of admissible functions as we have already noted in (i) of the Introduction; for example, the condition (8) would

* The Euler-Maclaurin expansion for $\lambda=\frac{1}{2}$ is less well known. It was given by Maclaurin; see Enzyklopadie II C2 $\$ 9$, p. 91. 
exclude the function $g(x)=\exp \left(-x^{2}\right)$, yet Goodwin has shown (loc. cit.) that the infinite integral of this function can be evaluated with high accuracy by the trapezoidal sum for moderate values of $h$.

It is sufficient in numerical evaluation that the bound of $E$ be less than the prescribed error of the calculation. In general, then, we reject the condition (8) and the stronger form of (2) as unnecessarily restrictive.

We shall give at the end of Section 2.3 some illustrations of the uses of equations (4) and (5). Before doing so, we examine the possibility of evaluating the infinite integral by a sequence of transformations of the type (4).

2.2. Successive Summations. If the error term in (4) is bounded and negligible, the correction term on the right of this equation is a new integral which can be evaluated in the same manner as the first integral. This may appear to be a profitless manoeuvre but the range of the new integration is $(0, d)$ instead of $(0, \infty)$; moreover the effective range of the new trapezoidal sum may be even smaller; for example, with $\lambda=0$, it can be seen from the first of (6) that the integrand possesses the damping factor $\exp (2 \pi y / h)-1$ in the denominator. In these circumstances, we may employ a smaller interval in evaluating the correction term and the total number of ordinates will not be excessive. It is convenient here to modify our notation, replacing $h$ by $h_{1}$ and $d$ by $d_{1}$. Integrating now round the rectangular contour

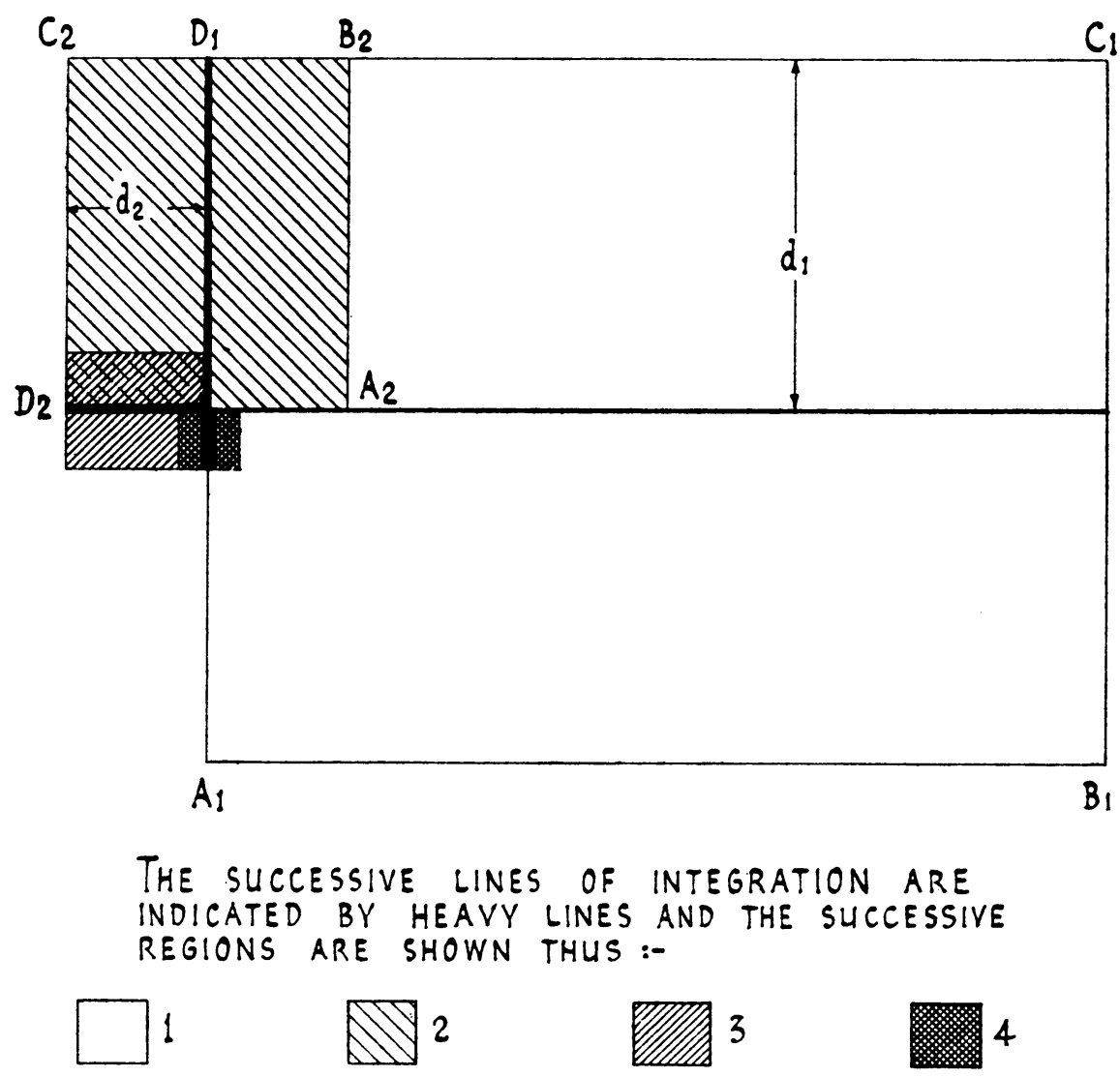

Fig. 1 
$A_{2} B_{2} C_{2} D_{2} A_{2}$ of Figure 1, the half-height of the rectangle being $d_{2}$ and the tabular interval $h_{2}\left(h_{2}<h_{1}\right)$, we have for $\lambda=0$

$$
\int_{0}^{\infty} g(x) d x-T_{1}-T_{2} \sim-i^{2} \int_{0}^{d_{2}} \frac{g\left(i^{2} x\right)-g\left(-i^{2} x\right)}{\exp \left(2 \pi x / h_{2}\right)-1} d x
$$

where

$$
\begin{aligned}
T_{1} & =h_{1}\left[\frac{1}{2} g(0)+g\left(h_{1}\right)+g\left(2 h_{1}\right)+\cdots\right], \\
T_{2} & =h_{2}\left[\frac{1}{2} G(0)+G\left(h_{2}\right)+G\left(2 h_{2}\right)+\cdots\right]
\end{aligned}
$$

and

$$
G(x)=-i \frac{g(i x)-g(-i x)}{\exp \left(2 \pi x / h_{1}\right)-1} .
$$

The damping factor of the new error term (i.e. the contribution from the lines $A_{2} B_{2}$ and $\left.C_{2} D_{2}\right)$ is exp $-\left(2 \pi d_{2} / h_{2}\right)$ and we assume that $d_{2}$ and $h_{2}$ can be chosen so that this error term is negligible. We assume also that the contribution from the line $B_{2} C_{2}$ is negligible.

If $m$ summations are carried out, and, if at each stage the contributions from the error terms and from lines such as $B_{2} C_{2}$ can be suitably bounded, we have

$$
\int_{0}^{\infty} g(x) d x-\sum_{1}^{m} T_{r} \sim-i^{m} \int_{0}^{d_{m}} \frac{g\left(i^{m} x\right)-g\left(-i^{m} x\right)}{\exp \left(2 \pi x / h_{m}\right)-1} d x .
$$

At this stage, it may be practicable to evaluate the remainder term on the right of (9) by an Euler-Maclaurin expansion. The process is illustrated diagrammatically in Figure 1 and it is evident that after each four summations we return to the real axis and begin a new cycle.

A similar iteration may be used if $\lambda=\frac{1}{2}$ and this modification enables us to deal with a singularity of the type $x^{a}(a>-1)$ or of the type $\log x$ at the origin.

If $g(x)$ is analytic within the successive regions of integration, we have under the same conditions as above

$$
\int_{0}^{\infty} g(x) d x-\sum_{1}^{m} M_{r}=i^{m} \int_{0}^{d_{m}} \frac{g\left(i^{m} x\right)-g\left(-i^{m} x\right)}{\exp \left(2 \pi x / h_{m}\right)+1} d x
$$

the character $M$ being used to indicate that the summations are mid-ordinate. When $g(x)$ is not analytic, the integral remaining after each summation does not possess an integrand of the simple form given in (10) as is evident from the examples of Section 2.3. When there is a singularity, the decreasing tabular interval introduces ordinates close to the singularity but no large numbers are introduced by reason of the factor $h_{r}$ which decreases more rapidly than the ordinates increase.

We quote here for completeness the principal formulae of trapezoidal and midordinate type, using the notation

$$
\begin{array}{rlrl}
I_{n} & =\int_{0}^{n h} g(x) d x=h \int_{0}^{n} g(p h) d p, & & \text { if } x=p h, \\
T_{n} & =\frac{1}{2} g_{0}+g_{1}+g_{2}+\cdots+g_{n-1}+\frac{1}{2} g_{n}, & \\
M_{n} & =g_{1 / 2}+g_{1(1 / 2)}+\cdots+g_{n-1 / 2} ; &
\end{array}
$$


then

$$
\begin{aligned}
I_{n}-h T_{n}=h & {\left[\begin{array}{l}
-\frac{1}{12}+\frac{1}{720}(h D)^{2}-\frac{1}{30240}(h D)^{4} \\
+\frac{1}{1209600}(h D)^{6}-\frac{1}{47900160}(h D)^{8}+\cdots
\end{array}\right] h D\left(g_{n}-g_{0}\right) } \\
= & h\left[\begin{array}{l}
-\frac{1}{12}+\frac{1}{720} \delta^{2}-\frac{1}{6720} \delta^{4} \\
+\frac{79}{3628800} \delta^{6}-\cdots
\end{array}\right] h D\left(g_{n}-g_{0}\right) \\
= & h\left[\begin{array}{l}
-\frac{1}{12}+\frac{11}{720} \delta^{2}-\frac{191}{60480} \delta^{4} \\
+\frac{2497}{3628800} \delta^{6}-\cdots
\end{array}\right] \mu \delta\left(g_{n}-g_{0}\right) .
\end{aligned}
$$

$D$ here denotes $d / d x$ and $\mu, \delta$ are the averaging and central-difference operators. Also

$$
\begin{aligned}
I_{n}-h M_{n} & =h\left[\begin{array}{c}
\frac{1}{24}-\frac{7}{5760}(h D)^{2}+\frac{31}{967680}(h D)^{4} \\
-\frac{127}{154828800}(h D)^{6}+\cdots
\end{array}\right] h D\left(g_{n}-g_{0}\right), \\
& =h\left[\begin{array}{c}
\frac{1}{24}-\frac{17}{5760} \delta^{2}+\frac{367}{967680} \delta^{4} \\
-\frac{27859}{464486400} \delta^{6}+\cdots
\end{array}\right] \delta\left(g_{n}-g_{0}\right) .
\end{aligned}
$$

It may be noted that the first term in the mid-ordinate formula is half the magnitude of the first term in the trapezoidal formula and is of opposite sign. This difference in sign is sometimes a useful check.

2.3. Control of the Error Term in Equation (4). There is no general method of bounding the error term $E(\lambda, h, d)$ in (4) but a few illustrations will show how the parameters $d$ and $h$ can be chosen. The discussion is simplified if we notice that the denominator of the integrand in the error term can be replaced by unity for all practical values of the ratio $d / h$.

When $g(x)$ is even and analytic, the discussion is considerably simplified and we shall consider this case first. Inspection of (4) shows that the correction term is then zero for $\lambda=0, \frac{1}{2}$. The left side of (4) is independent of $d$ and the right side must also be independent; i.e. when $g(x)$ is even, all finite choices of $d$ yield the same error term. We may then use any convenient technique, such as minimizing the absolute value of the integrand, to bound the error term.

For a small class of even functions $g(x)$, trapezoidal or mid-ordinate integration is exact for quite large values of $h$. This class is defined by the conditions $(2)$ and (8); e.g. the infinite integrals of $\sin x / x$ and of $J_{n}(x) / x^{m}[n>0, n-m$ positive 
even or zero] can be evaluated exactly for any $h$ which is less than $2 \pi$. When $g(x)$ is not even, the correction term $C(\lambda, h, d)$ must be computed but we can often carry out the computations by forming successive trapezoidal or mid-ordinate sums.

To illustrate the choice of $d$ when the error-term does not vanish, we consider in turn $g(x)=\exp \left(-x^{4}\right), A(x) e^{-a x^{2} \pm m i x}$ where $|A(z)|$ is bounded, $|z| \rightarrow \infty$. The latter type of function arises frequently in numerical work.

(i) $g(x)=\exp \left(-x^{4}\right)$.

On $y=d$, we have

$$
\left|\exp \left(-z^{4}\right)\right|=\exp -\left(x^{4}-6 x^{2} d^{2}+d^{4}\right)
$$

and the least value of the argument in parentheses occurs for $x^{2}=3 d^{2}$ and is $-8 d^{4}$. We determine $d$ by maximizing $2 \pi d / h-8 d^{4}$ : i.e. $d=(\pi / 16 h)^{1 / 3}$ and

$$
\exp -\left(2 \pi d / h-8 d^{4}\right)=\exp -(3 \pi d / 2 h) ;
$$

for $h \leqq \frac{1}{4}$ we can obtain very high accuracy.

(ii) $g(x)=A(x) e^{-a x^{2} \pm m i x}$.

We suppose that $|A(z)|$ is bounded. It is then obvious that

$$
|g(x \pm i d)| \leqq|A| \exp -\left(a x^{2}-m d-a d^{2}\right) .
$$

Here we maximize $2 \pi d / h-m d-a d^{2}$, obtaining $d=(\pi / h-m / 2) / a$; the error term of the integral over $(0, \infty)$ is then

$$
\leqq \sqrt{\frac{\pi}{a}}\left[\exp \left(-a d^{2}\right)\right]
$$

If $g(x)$ is not even, the correction term must be evaluated by a new trapezoidal or mid-ordinate sum or by one of the central difference formulae quoted earlier.

It is worth recalling that the choice of $d$ in the above illustrations has no effect on the actual value of the error term; it merely enables us to obtain a satisfactory practical bound on the error term.

EXAMPLE 1.

$$
\int_{0}^{\infty} \frac{\operatorname{erf} x \operatorname{erf} \lambda x}{x^{2}} d x
$$

where

$$
\operatorname{erf} x=\frac{2}{\sqrt{ } \pi} \int_{0}^{x} e^{-t^{2}} d t
$$

In this example, only limited precision is possible. The error term is

$$
0\left[\exp -\left(\pi^{2} /(1+\lambda) h^{2}\right)\right] .
$$

When $\lambda=1$, the error expected for $h=1, \frac{1}{2}$ is

$$
\begin{array}{ll}
|E| \leqq 0\left(e^{-\pi^{2} / 2}\right)=0\left(10^{-2}\right), & h=1, \\
|E| \leqq 0\left(e^{-2 \pi^{2}}\right)=0\left(10^{-8}\right), & h=\frac{1}{2} .
\end{array}
$$

Using $6 \mathrm{D}$ ordinates for $h=1$ and 10D ordinates for $h=\frac{1}{2}$, we obtain

$$
\begin{array}{lll}
1.989361 & 1.9890471882
\end{array}
$$

and the latter result is correct to $9 \mathrm{D}$. 
Example 2.

$$
J_{n}(\lambda)=\frac{1}{\pi} \int_{0}^{\pi} \cos (n x-\lambda \sin x) d x, \quad n, \text { integer; } \lambda>0 .
$$

In this example, the error term is not easily dominated and it serves as a useful illustration. There are more efficient ways of computing $J_{n}(\lambda)$ for large $n$ and $\lambda$; but we are here interested in obtaining an error bound.

For integer $n$, the correction terms are zero since the integrand is an even function of $y$ at both ends of the range. The error term is

$$
E=-\frac{2}{\pi} R \int_{0}^{\pi} \frac{\cos [n(x+i b)-\lambda \sin (x+i b)]}{\exp 2 \pi(b-i x) / h-1} d x
$$

where we have temporarily replaced $d$ (the half-height of the rectangular contour) by $b$ to avoid a notational confusion below; since

$$
|\cos [n(x+i b)-\lambda \sin (x+i b)]| \leqq \cosh (n b+\lambda \sinh b)
$$

we have

$$
|E| \leqq 2 e^{-2 \pi b / h} \frac{\cosh (n b+\lambda \sinh b)}{1-\exp -(2 \pi b / h)} .
$$

If $b / h$ is large, we may replace the denominator by unity and we find after a little manipulation

$$
|E|<\frac{h}{\pi} \frac{d u}{d b} \exp \left(-\frac{2 \pi b}{h}\right) \sinh u
$$

where $u=n b+\lambda \sinh b$ and $b$ is determined by

$$
\frac{2 \pi}{h}-\frac{d u}{d b} \tanh u=0 .
$$

For practical values of $u, \tanh u$ is very nearly unity and we can deduce from (11) the inequality

$$
h \leqq \frac{2 \pi}{n+\lambda \cosh b} .
$$

E.g. for $n=5, \lambda=5, h=\pi / 10$

$$
b \doteq 1.763 \text { and } E \doteq 10^{-6}
$$

or, for $n=5, \lambda=20$

$$
\begin{array}{lll}
\text { with } & h=\pi / 20, & |E|<10^{-5}, \\
\text { with } & h=\pi / 25, & |E|<10^{-10} .
\end{array}
$$

EXAmple 3.

$$
I=\int_{0}^{\infty} \frac{\sin x}{1+x^{2}} d x
$$

The error term is zero here for $h<2 \pi$. If we employ a trapezoidal sum $T_{1}$ with 
interval $h_{1}$, we have

$$
I-T_{1}=2 \int_{0}^{\infty} \frac{\sinh x d x}{\left(1-x^{2}\right)\left[\exp \left(2 \pi x / h_{1}\right)-1\right]}
$$

with $h_{1}=\pi$, the trapezoidal sum $T_{1}$ is zero, and we exchange an integrand which alternates in sign every half-period of $\sin x$ for a positive integrand $(x>1)$ which decreases as exp $(-x)$; there would be little profit or loss in the exchange. With a smaller value of $h_{1}$, it is convenient to sum $T_{1}$ by Eulering and this requires that we use an integer number of half-periods; with $h_{1}=\pi / 4$ (3 ordinates in each halfperiod), the integrand of $I-T_{1}$ decreases as $\exp (-7 x)$. A second sum $T_{2}$ with interval $h_{2}$ yields

$$
I-T_{1}-T_{2}=-2 \int_{0}^{\infty} \frac{\sin x d x}{\left(1+x^{2}\right)\left[\exp \left(2 \pi x / h_{2}\right)-1\right]}
$$

and it is now practical to employ the Euler-Maclaurin series with $h_{2}=\frac{1}{8}$ or $\frac{1}{16}$. Equivalent accuracy would be obtained by using $h_{1}=\frac{1}{8}$ or $\frac{1}{16}$ without employing $T_{2}$; but $T_{1}$ is then more difficult to evaluate.

\section{Error Bounds for Gaussian Integration.}

3.1. Gauss-Legendre. The Gauss-Legendre formula is a convenient startingpoint. Denoting the zeros of the Legendre polynomial $P_{n}(t)$ by $t_{i}$ and the corresponding weights by $h_{i}$, we begin by expressing the difference

$$
\int_{-1}^{1} g(t) d t-\sum_{i} h_{i} g\left(t_{i}\right)
$$

as a contour integral and then use this contour integral to obtain bounds.

If $L$ is any simple contour enclosing the strip $(-1,1)$ in the $z$-plane, we can deduce from the contour integral

that

$$
\frac{1}{2 \pi i} \int_{L} \frac{g(z) d z}{(z-t) P_{n}(z)}
$$

$$
g(t)=P_{n}(t) \sum_{i} \frac{g\left(t_{i}\right)}{\left(t-t_{i}\right) P_{n}^{\prime}\left(t_{i}\right)}+\frac{1}{2 \pi i} \int_{L} \frac{g(z) P_{n}(t)}{(z-t) P_{n}(z)} d z
$$

if $g(z)$ is regular within $L$.

If we integrate this equation with respect to $t$ over $(-1,1)$ and interchange the order of integration in the repeated integral on the right, we obtain

$$
\int_{-1}^{1} g(t) d t=\sum_{i} \frac{g\left(t_{i}\right)}{P_{n}^{\prime}\left(t_{i}\right)} \int_{-1}^{1} \frac{P_{n}(t)}{t-t_{i}} d t+\frac{1}{2 \pi i} \int_{L} \frac{g(z) d z}{P_{n}(z)} \int_{-1}^{1} \frac{P_{n}(t)}{z-t} d t .
$$

We can now employ two known results. If $z$ is not a real number between -1 and +1 , unless it be a zero of $P_{n}(t)$, then

$$
2 Q_{n}(z)=\int_{-1}^{1} \frac{P_{n}(t)}{z-t} d t
$$

* The singularity at $x=1$ in $I-T_{1}$ requires a slight modification of our earlier procedure; this modification is assumed to be absorbed in $T_{2}$. 
where $Q_{n}(z)$ is the Legendre function of the second kind. We also have

$$
Q_{n}(z)=\frac{1}{2} P_{n}(z) \log \frac{z+1}{z-1}-f_{n-1}(z)
$$

where $f_{n-1}(z)$ is a polynomial of order $n-1$ (the coefficients of $f_{n-1}$ are tabulated in Whittaker and Watson [10]). Using these results, we can write (13) as

$$
\int_{-1}^{1} g(t) d t-\sum h_{i} g\left(t_{i}\right)=\frac{1}{\pi i} \int_{L} \frac{g(z) Q_{n}(z)}{P_{n}(z)} d z
$$

the weights $h_{i}$ being determined by

$$
h_{i}=\frac{2 f_{n-1}\left(t_{i}\right)}{P_{n}^{\prime}\left(t_{i}\right)}
$$

but they need not concern us here because they have been extensively tabulated.

The practical use of the error term on the right of (14) depends on the fact that all contours $L$ which enclose the zeros of $P_{n}(z)$ are equivalent if we assume that $g(z)$ is regular in a sufficiently large domain (clearly, it would not be difficult to take into account a finite number of poles of $g(z)$ and we show this briefly at the end of this section). It is convenient to choose as contour a circle of sufficiently large radius $R$ to permit the use of the asymptotic value, $|z| \rightarrow \infty$, of $Q_{n}(z) / P_{n}(z)$ :

$$
\frac{Q_{n}(z)}{P_{n}(z)}=\frac{2^{2 n}(n !)^{4}}{(2 n) !(2 n+1) !} z^{-2 n-1}\left[1+\frac{2 n^{3}+3 n^{2}-n-1}{(2 n+3)(2 n-1) z^{2}}+0\left(z^{-4}\right)\right] \text {. }
$$

As a simple illustration, consider

$$
g(t)=e^{a t} t^{m}, \quad m \text {, integer. }
$$

Taking only the first term of the asymptotic expansion for $Q_{n}(z) / P_{n}(z)$, the modulus of the integrand in the error term is dominated by

$$
C \exp \{-[(2 n-m) \log R-a R]\}
$$

on a large circle of radius $R$, the factorial constant above being denoted by $C$. The least value of the expression in brackets, qua function of $R$, is

$$
C \exp -[a R(\log R-1)], \quad a R=2 n-m,
$$

and the error $E$ of Gaussian integration is given by

$$
|E| \leqq 2 C \exp -[a R(\log R-1)]
$$

e.g. if $m=3, a=1$, we have

$$
\begin{array}{ll}
n=5, & |E| \leqq 4 \times 10^{-6} \\
n=6, & |E| \leqq 2 \times 10^{-9}, \\
n=7, & |E| \leqq 4 \times 10^{-11} .
\end{array}
$$

These error bounds could be improved by a more careful analysis but they are 
simply obtained and they are not unduly pessimistic, the actual errors being

$$
n=5: \quad 1.0 \times 10^{-7} \quad n=6: 2.1 \times 10^{-10} \quad n=7: 4.8 \times 10^{-12} .
$$

If $g(z)$ is a meromorphic function, the contribution of the residues at the poles of $g(z)$ to the right side of (12) is some function of $t, p(t)$ say, and (14) is then replaced by

$$
\int_{-1}^{1} g(t) d t-\sum_{i} h_{i} g\left(t_{i}\right)-\int_{-1}^{1} p(t) d t=\frac{1}{\pi i} \int_{L} \frac{g(z) Q_{n}(z)}{P_{n}(z)} d z
$$

and the error term may be estimated as above. The right side of (16) vanishes and the integration formula is exact if $g(z)$ is a meromorphic function and if

$$
|g(z)| \leqq 0\left(|z|^{2 n-1}\right), \quad|z| \rightarrow \infty
$$

uniformly with respect to the argument of $z$. We assume here that $g(z)$ is such that the integrals in (16) exist.

For example, if $g(z)$ satisfies the above inequality and has simple poles at the points $a_{k}$ with residues $A_{k}$, then from (16)

$$
\int_{-1}^{1} g(t) d t=\sum_{i} h_{i} g\left(t_{i}\right)-2 \sum_{k} A_{k} \frac{Q_{n}\left(a_{k}\right)}{P_{n}\left(a_{k}\right)}
$$

3.2. Gauss-Laguerre and Gauss-Hermite Formulae. The method used in Section 3.1 is applicable here but some modification is needed since the range of integration is infinite in the Gauss-Laguerre and Gauss-Hermite formulae. We consider GaussLaguerre in some detail; the analysis for Gauss-Hermite is similar and may be omitted.

Supposing $g(z)$ to be regular within a contour $L$ which encloses all the zeros of the Laguerre polynomial $L_{n}(z)$, we can write

$$
g(t)=\sum_{i} \frac{g\left(t_{i}\right) L_{n}(t)}{\left(t-t_{i}\right) L_{n}{ }^{\prime}\left(t_{i}\right)}+\frac{1}{2 \pi i} \int_{L} \frac{g(z) L_{n}(t)}{(z-t) L_{n}(z)} d z,
$$

the summation on the right being effected over the zeros $t_{i}$ of $L_{n}(t)$. It is convenient at this point to take for contour the circle $z=R_{1} e^{i \theta}$. We now multiply (17) by $e^{-t}$ and integrate with respect to $t$ over $(0, R)$, interchanging the order of integration in the double integral. We obtain

$$
\begin{aligned}
\int_{0}^{R} e^{-t} g(t) d t-\sum_{i} & \frac{g\left(t_{i}\right)}{L_{n}{ }^{\prime}\left(t_{i}\right)} \int_{0}^{R} \frac{e^{-t} L_{n}(t)}{t-t_{i}} d t \\
& =\frac{1}{2 \pi i} \int \frac{g(z)}{L_{n}(z)} d z \int_{0}^{R} \frac{e^{-t} L_{n}(t)}{z-t} d t, \quad R<R_{1} .
\end{aligned}
$$

In the limit, $R, R_{1} \rightarrow \infty$, we can write

$$
\int_{0}^{\infty} e^{-t} g(t)-\sum_{i} H_{i} g\left(t_{i}\right)=E
$$

where the $H_{i}$ are the Laguerre weights and the error term, $E$, is defined by

$$
E=\lim _{R \rightarrow \infty ; R_{1} \rightarrow \infty} \frac{1}{2 \pi i} \int_{L} \frac{g(z)}{L_{n}(z)} d z \int_{0}^{R} \frac{e^{-t} L_{n}(t)}{z-t} d t
$$


From the analysis of the Gauss-Legendre formula, we anticipate that the error term will vanish if

$$
|g(z)| \leqq 0\left(|z|^{2 n-1}\right) \quad|z| \rightarrow \infty, 0 \leqq \theta \leqq 2 \pi .
$$

To show this, we split the error term into two parts $E_{1}$ and $E_{2}$ by expanding the denominator $z-t$ in $(20)$ :

and

$$
E_{1}=\lim \frac{1}{2 \pi i} \int_{L} \frac{g(z)}{L_{n}(z)} \sum_{m=0}^{n} \frac{d z}{z^{m+1}} \int_{0}^{R} e^{-t} t^{m} L_{n}(t) d t
$$

$$
E_{2}=\lim \frac{1}{2 \pi i} \int_{L} \frac{g(z) d z}{z^{n+2} L_{n}(z)} \int_{0}^{R} \frac{e^{-t} t^{n+1} L_{n}(t)}{(1-t / z)} d t .
$$

Considering $E_{1}$ first, we may write

$$
\sum_{m=0}^{n} \frac{1}{z^{m+1}} \int_{0}^{R} e^{-t} t^{m} L_{n}(t) d t=\sum_{m=0}^{n} \frac{1}{z^{m+1}} \int_{0}^{\infty} e^{-t} t^{m} L_{n}(t) d t+\epsilon(n, R)
$$

and the epsilon term approaches zero, $R \rightarrow \infty$. On using the orthogonal property of the Laguerre polynomials, only the term $m=n$ survives in the summation on the right; we have

$$
\left|E_{1}\right| \leqq \frac{1}{2 \pi} \mid \lim _{R \rightarrow \infty ; R_{1} \rightarrow c}\left[(-)^{n}(n !)^{2}+\epsilon(n, R)\right] \int_{L} \frac{g(z) d z}{z^{n+1} L_{n}(z)}
$$

and $\left|E_{1}\right| \rightarrow 0$ if (21) is satisfied.

To bound $E_{2}$, it is convenient to write

$$
R_{1}=e^{R} \quad \text { where } \quad z=R_{1} e^{i \theta}
$$

the inner integral in $E_{2}$ can now be written as

$$
\int_{0}^{R} \frac{e^{-t} t^{n+1} L_{n}(t)}{1-t e^{-R-i \theta}} d t=\int_{0}^{R} e^{-t} t^{n+1} L_{n}(t) d t+e^{-R-i \theta} \int_{0}^{R} \frac{e^{-t} t^{n+2} L_{n}(t)}{1-t e^{-R-i \theta}} d t
$$

and the second integral on the right approaches zero, $R \rightarrow \infty$. Since the first integral on the right is bounded, it follows that $\left|E_{2}\right|$ vanishes if $g(z)$ is an entire function satisfying (21), i.e., if $g(z)$ is a polynomial of degree not greater than $2 n-1$.

We may obviously extend the analysis to meromorphic functions which satisfy (21). Modifying (17) to take account of the poles of $g(z)$, we have in the notation of (16)

$$
\int_{0}^{\infty} e^{-t} g(t) d t=\sum_{i} H_{i} g\left(t_{i}\right)+\int_{0}^{\infty} e^{-t} p(t) d t
$$

Conclusion. The error of the $n$-point Gauss-Legendre procedure depends on $n$ and also on the function to be integrated. It would be useful if we had some estimate of the dependence on $n$.

The results obtained above for the Gauss-Legendre case permit a conjecture. If we use the Stirling approximation for the factorial terms in the expression (15) for $Q_{n}(z) / P_{n}(z)$ and retain only the dominant term, we have 


$$
\frac{Q_{n}(z)}{P_{n}(z)} \sim(2 z)^{-2 n-1} \pi
$$

for large $n$ and $|z|(|z|>n)$. For the function $g(z)=\exp (a z)$, Section 3.1 yields the error estimate

$$
E_{n} \leqq 2 \pi \exp -\left[(2 n+1) \log 2+2 n\left(\log \frac{2 n}{a}-1\right)\right]
$$

A similar expression for $E_{n+1}$ is obtained if we replace $n$ by $n+1$. We conjecture that an estimate of the ratio $E_{n+1} / E_{n}$ may be obtained by taking the ratio of the corresponding expressions, i.e.,

$$
\frac{E_{n+1}}{E_{n}}=O\left(\frac{a}{4 n+4}\right)^{2}
$$

It does not appear easy to confirm or disprove this conjecture.

National Bureau of Standards

Washington 25, D. C.

University of Alberta

Edmonton, Alberta, Canada

1. E. T. Goopwin, "On the evaluation of integrals of the form $\int_{-\infty}^{\infty} \exp \left(-x^{2}\right) f(x) d x$," Proc. Cambridge Philos. Soc., v. 45, 1949, p. 241-245.

2. P. Davis, On the Numerical Integration of Periodic Analytic Functions, Symposium on Numerical Approximation, Wisconsin, 1959, p. 45-59.

3. E. Lindelöf, Le Calcul Des Résidus, Chelsea, New York, 1947.

4. G. H. Hardy, Divergent Series, Oxford University Press, Oxford, 1949.

5. , Modern Computing Methods, H. M. Stationery Office, London, 1961.

6. H. E. FetTIs, "Numerical calculation of certain definite integrals by Poisson's Summation Formula," Math. Comp., v. 9, 1955, p. 85-92.

7. A. CaUCHY, "Mémoire sur les développements des fonctions en séries périodiques," Mémoires de l'Institut, v. 6, 1826, p. 603-612.

8. G. Plana, "Sur une nouvelle expression analytique des nombres bernoulliens," Academia di Torino, v. 25,1820 , p. 403-418.

9. N. H. ABEL, "Solution de quelques problèmes à l'aide d'integrales définies," Oeuvres complètes D'Abel, v. 1, p. 11-27.

10. E. T. Whittaker \& G. N. Watson, Modern Analysis, Cambridge University Press, Cambridge, 1920. 